support in adults'. Patients' notes were then recalled following discharge to determine whether further nutritional interventions had taken place during their admission.

Results We found that only $36 \%$ of patients were screened appropriately for malnutrition and through our analysis, we identified that at admission $51 \%$ were either malnourished or at risk of malnutrition. Only one patient was referred to a dietician and started on nutritional support during the acute admission period.

Conclusion Nutrition assessment needs to be reinforced as a clinical priority and essential screening for patients admitted to hospital through the Acute Medical Unit. This should be jointly addressed by the medical and nursing teams and by ensuring early input from dieticians is available.

Disclosure of Interest None Declared.

\section{PTH-022 SAFETY AND EFFICACY OF DAY CASE THERAPEUTIC PARACENTESIS FOR MALIGNANT ASCITES}

A Xiarchou*, BR Disney, C Hammond, E Sung, S Raman, GM Wood. Gastroenterology, George Eliot Hospital, Nuneaton, UK

\subsection{6/gutjnl-2014-307263.468}

Introduction Ascites is a common consequence of cancer, occurring in $15-50 \%$ of patients, and causes symptoms such as abdominal discomfort, anorexia, nausea and a reduced quality of life. Therapeutic paracentesis is the most often utilised and the most effective treatment for malignant ascites. It can improve the patient's symptoms and quality of life, and has a low risk of complications. The aim of this study was to assess the safety and efficacy of day case paracentesis in the context of malignant ascites.

Methods We performed a retrospective analysis of patients with malignant ascites admitted as a day case to our Ambulatory Care Unit (ACU) for therapeutic paracentesis. Drains were placed without ultrasound guidance by doctors experienced in the procedure. Drains were left in situ for a maximum of $6 \mathrm{~h}$. Albumin or fluids were not given. Data on blood pressure and heart rate before and after the procedure, the volume of fluid removed, duration of stay, any complications and any fluids given, were recorded. Data such as age, albumin levels, having chemotherapy at that time and if they are still alive were collected for all the patients.

Results A total of 20 ascitic drains, performed in 9 patients, were identified. Of these, 19 drains were performed successfully as day-case procedures at ambulatory care unit (ACU).

Mean age of patients was 75 years (range $45-85$ years). All patients had cancer of gastrointestinal origin. Five patients were having chemotherapy at the time of the procedure. The average albumin was $31.3 \mathrm{~g} / \mathrm{L}$ (range 23-41).

The mean volume of ascites drained was $8.3 \mathrm{~L}$ (range 4.0$14.8 \mathrm{~L})$.

There were no significant changes in either mean arterial pressure or heart rate before and after paracentesis. Heart rate: before $82 \pm 18 \mathrm{bpm}$, after $82 \pm 13 \mathrm{bpm}(\mathrm{p}=0.95)$. Mean arterial pressure: before $91 \pm 10 \mathrm{mmHg}$ and after $84 \pm 11 \mathrm{mmHg}$ $(\mathrm{P}=0.07)$.

The maximum time of staying in ACU was $8.5 \mathrm{~h}$ (mean time $<7 \mathrm{~h}$ ). Six patients have since died from cancer related deaths.

There was one failed paracentesis; this patient subsequently underwent uncomplicated ultrasound guided placement. One drain was delayed by $48 \mathrm{~h}$ due to neutropenia. There were no serious complications and no patients required hospital admission. One minor complication was reported (skin haematoma) and one patient required intravenous fluids.
Conclusion Day case paracentesis is both safe and effective in the management of malignant ascites. It is not necessary to routinely replace fluid or albumin during the procedure. This approach can avoid unnecessary hospital admissions and provides rapid improvement in patient's symptoms and patient quality of life.

Disclosure of Interest None Declared.

\section{PTH-023 PAEDIATRIC FAECAL VOC ANALYSIS: METHOD OPTIMISATION}

A Mayor*, S Reade, R Aggio, T Khalid, C Probert. Gastroenterology, University of Liverpool, Liverpool, UK

\subsection{6/gutjnl-2014-307263.469}

Introduction Faecal Volatile Organic Compounds (VOCs) analysis is an emerging diagnostic tool for gastrointestinal conditions because of its sampling ease and non-invasive characteristics. Solid phase micro-extraction (SPME) is often used with gas chromatography-mass spectrometry for the analysis of VOCs; however no procedure has been standardised for an application in faecal analysis with the potential for on-site utilisation. Several aspects of the sampling preparation applied to neonatal faecal samples were studied to improve the robustness of the analytical process for paediatric studies.

Methods Thirty-three faecal neonate samples of weight 50-700 mg were analysed. The results produced by SPME coatings CAR/PDMS and DVB/CAR/PDMS were compared ( $\mathrm{n}=5 /$ variable), as were vial volumes of 2 and $10 \mathrm{ml}(\mathrm{n}=4 /$ variable; $\mathrm{N}=3$ ) and the addition of 0.5 and $1 \mathrm{ml}(\mathrm{n}=3 /$ variable $)$ of a saturated $\mathrm{NaCl}$ solution prior to GC-MS analysis. In addition, the influence of leaving the samples for $14 \mathrm{~h}$ at $1{ }^{\circ} \mathrm{C}$ instead of $-20^{\circ} \mathrm{C}$ was studied ( $n=3 /$ condition). Finally, the reproducibility of the method was tested by looking at the internal variation of 10 sets of triplicate; furthermore, 3 sets were reanalysed 4 times in order to characterise the repeatability across GC-MS runs. Independent samples t-test, one-way ANOVA and Tukey's HSD test were performed to test differences between data classes. Final pvalues were adjusted by Bonferroni and p-values $<0.05$ were considered significant.

Results Twenty $( \pm 2)$ VOCs were identified using samples of 100 $\mathrm{mg}$, while $28( \pm 1)$ and $25( \pm 2)$ VOCs were identified in samples of 450 respectively $700 \mathrm{mg}$. There were no significant differences in VOCs intensities between samples of 450 and $700 \mathrm{mg}$ as between 50 and $100 \mathrm{mg}$. However, all VOCs intensities were significantly higher in samples of 450 and $700 \mathrm{mg}$ when compared to $100 \mathrm{mg}$. No differences were observed between the two SPME coatings, and the addition of salt did not improve results quantitatively or qualitatively. Keeping samples at $1^{\circ} \mathrm{C}$ instead of $-20^{\circ} \mathrm{C}$ and/or varying the volume of the vial did not influence the results significantly. Finally, for 7 sets of triplicates out of 10 , more than $90 \%$ of the ion intensities varied less than $30 \%$ but multiple runs of GC-MS resulted in significant changes in intensity of $40 \%$ or more of the VOCs identified.

Conclusion Several parameters were studied to optimise a method for paediatric faecal VOCs analysis and a robust method has now been developed and detailed. Samples of $50-100 \mathrm{mg}$ may be studied. This weight gives reproducible results, but samples should not be reanalysed as headspace VOCs are altered by the procedure. Other changes to sample processing had little impact on the results. A chilled auto sampling rack may be safely used.

Disclosure of Interest None Declared. 